\title{
Analysis of the Polarization Distribution in a Polar Perhydrotriphenylene Inclusion Compound by Scanning Pyroelectric Microscopy
}

\author{
Andrea Quintel and Juirg Hulliger* \\ Department of Chemistry and Biochemistry, University of Berne, Freiestrasse 3, CH-3012 Berne, Switzerland
}

\author{
Michael Wuibbenhorst \\ Department of Polymer Technology, Delft University of Technology, P.O. Box 5045, 2600 GA Delft, \\ The Netherlands
}

Received: October 15, 1997; In Final Form: April 1, 1998

\begin{abstract}
The polarization distribution in a polar perhydrotriphenylene (PHTP) inclusion compound has been studied by scanning pyroelectric microscopy. Measurements have been performed on solution-grown crystals of PHTP-AD (AD: acceptor (A) and donor (D) disubstituted linear chromophores). The AD guest molecules form aligned dipole chains within the parallel channels created by the honeycomb-type host lattice of PHTP. Along the channel direction we found a $180^{\circ}$ twinned macrodomain state with wedge-shaped domain boundaries adjacent to the middle of the needle-shaped crystals. In each domain the polarization is nearly constant, which implies that the majority of molecular dipoles are aligned parallel. This agrees well with predictions arising from the application of Markov's theory of stochastic processes, which explains the occurrence of polar domains due to a mechanism of dipole incorporation driven by the difference in the $-\mathrm{A} \cdots \mathrm{A}-$ and $-\mathrm{D} \cdots \mathrm{D}-$ intermolecular interaction energies.
\end{abstract}

\section{Introduction}

Perhydrotriphenylene (PHTP) and A (acceptor), D (donor) disubstituted nonlinear optical (NLO) molecules (AD) form channel-type inclusion compounds. ${ }^{1-3}$ Dipolar guest molecules are close-packed along parallel channels built up by a honeycomblike stacking of PHTP. Second harmonic generation (SHG) experiments on more than 30 different PHTP-AD inclusions revealed macroscopic polar properties. ${ }^{3}$ Recently, we demonstrated $^{4,5}$ that this high incidence of polar supramolecular crystal structures is due to a crystal growth mechanism which can be described by a set of Markov probabilities for the attachment of $\mathrm{AD}$ dipoles to $\mathrm{A}$ and $\mathrm{D}$ surface sites. The theory of functional group attachment predicts a hourglass-like domain structure where polarity evolution results in two opposing macrodomains.

The subject of the present paper is to investigate the domain structure of two different PHTP-AD materials by means of a technique called scanning pyroelectric microscopy (SPEM). ${ }^{6-8}$ A combination of position-dependent thermal excitation (2-D scanning) with a controlled variation of the thermal penetration depth allowed us to obtain information on both the magnitude and direction of the polarization distribution in three dimensions.

A much simpler technique using charged powder particles was previously applied to organic crystals. ${ }^{9}$ In our case the use of $\mathrm{Pb}_{3} \mathrm{O}_{4}$ and sulfur powders led to a polarity assignment in agreement with SPEM.

\section{3-D Information from the Pyroelectric Response}

The polarization of a polar material can be considered by assuming an equivalent surface charge density $\sigma=\mathrm{d} P(x) / \mathrm{d} x$. Under ambient conditions the total electric field of these polarization charges is compensated by free real charges. When the temperature is changed by $\Delta T$, the polarization changes as
$\Delta P$, provided the pyroelectric coefficient $p=\mathrm{d} P / \mathrm{d} T$ is sufficiently large. The resulting change in the surface charge density may be measured as a change of the surface potential or as a discharge current. According to Figure 3 we adopted a current detection method that ensures operation under zero-field conditions.

With respect to the rate of temperature change, one usually distinguishes between quasistatic and dynamic pyroelectric techniques. Whereas the quasistatic techniques utilize temperature variations under nearly thermal equilibrium conditions, dynamic pyroelectric methods use fast temperature changes caused by (i) a pulsed or (ii) a periodically modulated heat flux. ${ }^{10,11}$ By employing 2-D SPEM, we developed a technique whereby a needle-like crystal is scanned in two dimensions by a focused laser beam of a sinusoidally modulated intensity. Partial absorption of radiation power causes periodical temperature changes (thermal waves) in a sample volume. This generally comprises a certain volume where the heat is generated as well as a region to which heat is spread out due to thermal diffusion.

In our study, a sinusoidally modulated laser diode was used as the heat source. Scanning was provided by a motorized biaxial positioning system. 1-D probing of the local pyroelectric effect in pyroelectric materials has been reported previously: 7,8 Yilmaz et al. applied SPEM to poled polymer films, ${ }^{7}$ which displayed a line-shaped polarization pattern. Application of SPEM to the analysis of the polarization distribution in channels of $\mathrm{AlPO}_{4}-5$ loaded with NLO entities ${ }^{8}$ revealed the phenomenon of polarization inversion, as found here for PHTP-AD crystals. However, the mechanism of in-diffusion of NLO molecules into channels of a zeolite differs considerably from that for cocrystallization. The first 2-D imaging of local pyroelectricity was reported in 1976 by Hadni et al. ${ }^{6}$ for triglycine sulfate, which 
has a 3 orders of magnitude higher pyroelectric coefficient than the inclusion compounds reported here.

Since we aim to know both the magnitude and the sign of the polarization, we have to specify models for (i) the propagation of heat into the crystal and (ii) the origin of the pyroelectric effect. Owing to the property of one-dimensional thermal waves, i.e. that the penetration length obeys $\lambda_{\text {th }}=\sqrt{K / \pi f}(K=$ thermal diffusivity, $f=$ modulation frequency), one can control the probe depth by tuning the frequency of laser modulation.

Two basic types of experiments were carried out: (i) measurement of pyroelectricity near the surface with a fixed thermal penetration length (SPEM-images); (ii) probing the polarization in the thickness direction of the sample by varying the frequency (pyroelectric spectra).

It will be shown below that these two basic experiments provide information about the 3-D polarization distribution. Using an appropriate model for the pyroelectric response, it should be possible to reconstruct the spatial polarization distribution $P(x, y, z)$ over the entire volume of the sample (tomography).

Generally, the pyroelectric response of a dielectric to a timedependent temperature field involves both thermal and electrostatic properties. Most of the pyroelectric techniques based on thermal waves start from the assumption of a thin sample in which the polarization and heat flow direction are parallel to the plane normal to the thickness direction ( $x$-axis). The temperature distribution then reduces to a one-dimensional $T$-profile $T(x, t)$, whereas the electric field between the two electrodes of the sample is homogeneous.

Using the substitution $P(x)=P^{*}(x)+\bar{P}$ (separation polarization distribution from its mean value), the complex amplitude of the pyroelectric current $I \sim$ is given by eq $1:{ }^{12}$

$I_{\sim}(\omega)=\left(\alpha_{\mathrm{p}}+\alpha_{1}-\alpha_{\epsilon}\right) \frac{A}{l} \int_{0}^{l} P^{*}(x) \frac{\partial T(x, \omega)}{\partial t} \mathrm{~d} x+\alpha_{\mathrm{p}} \bar{P} \frac{\mathrm{d} \bar{T}}{\mathrm{~d} t} A$

where $\alpha_{p}, \alpha_{1}$, and $\alpha_{\epsilon}$ are the temperature coefficient of the polarization, the linear expansion coefficient, and the temperature coefficient of the permittivity $(\epsilon)$, respectively. $A$ is the heated area and $l$ is the thickness of the sample. Note that the classical pyroelectric coefficient $p$ is represented by the second term according to $p=\alpha_{\mathrm{p}} \bar{P}$. It will be shown below that in most of the PHTP inclusion compounds, the mean polarization vanishes, and therefore the second term will be neglected. The remaining first expression, however, represents the deviation of the polarization from its mean value. In contrast to the homogeneous pyroelectric sample, here two additional material properties have to be taken into account $\left(\alpha_{1}\right.$ and $\left.\alpha_{\epsilon}\right)$.

It should be emphasized that, strictly speaking, eq 1 only holds for stress-free, i.e. unclamped, samples of constant area or samples where the electrodes follow the lateral thermal expansion ("soft electrodes"). When measuring crystals with a ratio $l / l_{3} \ll 1\left(l=l_{1}\right.$ or $l_{2}$, thicknesses in 1 - and 2-direction; $l_{3}$ : length of the sample in 3-direction), one has to adopt the assumptions made above.

2.1. Propagation of Heat. For the understanding of the pyroelectric response of a rod-like crystal, we have to be familiar with its 3-D heat transport behavior. For practical reasons it is always desirable to consider a 1-D heat flow in the length direction, as for a thin slab. Four special cases of simplified heat transport in a rod-shaped sample were considered (see Figure 1):

(1) The first is heating of a thin layer parallel to the cross section of the crystal by direct absorption of heat. In the case


Figure 1. Four special cases of heat transport in a needle-like sample between two plate electrodes: (a) heat absorption in the volume $\Longrightarrow$ $1-\mathrm{D}$ heat flow in the length direction of the needle; $(b-d)$ heat generation in a thin insulating absorption layer on the crystal surface. From high to low frequencies the heat flow direction changes from perpendicular to the needle axis to semispherical heat propagation (medium frequencies) to 1-D heat flow in the needle (3-) direction, similar to case (a).

of sufficiently low absorption and scattering, we obtain the situation shown in Figure 1a.

(2) Since unpolished crystals show strong scattering which obscures the well-defined local heating necessary for highresolution SPEM, one has to restrict heat generation to the crystal surface by use of a thin dielectric absorption layer. In this situation, heat flow changes drastically as indicated by Figure $1 b-d$.

Depending on the modulation frequency we distinguish the following:

(b) $\lambda_{\text {th }}<d$ (high frequencies, $10^{2}-10^{5} \mathrm{~Hz}$ ): 1-D heat flow perpendicular to the needle direction. The thermal penetration length is then characterized by $\sqrt{K_{i} / \pi f}$, with $i=1,2$. If we assume a thermal diffusivity, which is higher along the aligned dipole chains than perpendicular to them, we obtain: $K_{i} \ll K_{3}$.

(c) $d<\lambda_{\text {th }}<l_{1,2}$ (medium frequencies, $10 \mathrm{~Hz}$ ): In this case, semispherical heat propagation is expected.

(d) $\lambda_{\text {th }}>l_{1,2}$ (low frequencies, $<1 \mathrm{~Hz}$ ): again, 1-D heat flow in the 3 -direction. For crystals with a large aspect ratio the spatial extension of the zone close to the heating spot can be neglected and the quasi isothermally heated region is similar to case 1; see Figure 1a.

Case $2 b$ is interesting for surface SPEM: the penetration length is well-defined. Adjusting the spot size to $\lambda_{\text {th }}$ enables operation over a wide frequency range. In case $2 c$, the thermal resolution is worse than the optical resolution; that is, the heated surface region is significantly larger than the illuminated area. This means that the heat flow changes from a linear to a radial flow, resulting in a semispherical temperature distribution (isotropic thermal conductivity assumed). Since the thermal penetration length is the limiting factor for the spatial resolution of the SPEM, thermal conductivity can be estimated right away from the smoothness of the SPEM-image.

Summarizing, we can say that the thermal properties and the sample dimensions determine which particular heat transport model applies to a certain range of the modulation frequency. This influences the interpretation of the pyroelectric response since a particular heat flow direction as well as the contributing volume part both depend on the frequency $f$. For a given illuminated surface area this would mean that at high frequencies the lateral (1,2-direction) polarization distribution is probed, 
whereas at low frequencies the thermal wave front probes predominantly the polarization distribution along the 3 -axis.

2.2. Origin of the Pyroelectric Effect. The external electrical field of a material generated by its polarization is normally compensated at the surfaces by charges from its surroundings. Change of the temperature by $\Delta T$ disturbs this equilibrium situation and generates an electrical field change $\Delta E=\Delta P / \epsilon_{0} \epsilon$, which gives rise to a measurable change of the surface charge. To make a link between the measured charge $\Delta Q(t)$ and the local polarization, two issues have to be considered: (i) the molecular origin of the polarization and its temperature dependence (represented by the constant $\alpha_{\mathrm{p}}=p / P$ ); (ii) the relation between position-dependent polarization and the (measurable) electrode charge, the so-called response equation.

The molecular origin for the macroscopic polarization $P$ of the inclusion compounds is the arrangement of strong dipolar guest molecules (dipole moment $\mu$ ) in a non-centrosymmetric crystalline structure. Knowing the number of dipoles $N$ per unit volume $V$ as well as the mean deviation of the dipole axis from the crystal axis $\langle\cos \theta\rangle$, one can calculate the dipole moment per unit volume:

$$
P_{0}=\frac{N}{V} \mu\langle\cos \theta\rangle
$$

Since the elementary dipoles polarize their neighbors as well as the host lattice, the so-called Lorentz factor $L$ has to be introduced. The macroscopic polarization then reads

$$
P=[1+L(\epsilon-1)] P_{0}
$$

with $\epsilon$ being the dielectric constant of the crystal. $L$ is normally chosen to be $1 / 3$, which is strictly true only for dipoles in a cubic lattice, provided the lattice constant is much larger than the dipole length $l_{\mu}$. Although these two requirements are certainly not fulfilled by PHTP-guest systems, the approximation $L \sim 1 / 3$ is supported by numerous experimental work on NLO materials ${ }^{13}$ and will be used below.

The coefficient $\alpha_{\mathrm{p}}$ can be obtained by deriving $\alpha_{\mathrm{p}}=(1 / P)(\mathrm{d} P /$ $\mathrm{d} T$ ). After inserting eq 2 in eq 3 and using $V=A l$, we take the total derivative of $P$ :

$$
\begin{array}{r}
\frac{\mathrm{d} P}{\mathrm{~d} T}=\frac{\partial P}{\partial l} \frac{\mathrm{d} l}{\mathrm{~d} T}+\frac{\partial P}{\partial A} \frac{\mathrm{d} A}{\mathrm{~d} T}+\frac{\partial P}{\partial \mu} \frac{\mathrm{d} \mu}{\mathrm{d} T}+\frac{\partial P}{\partial\langle\cos \theta\rangle_{\mathrm{th}}} \frac{\mathrm{d}\langle\cos \theta\rangle_{\mathrm{th}}}{\mathrm{d} T}+ \\
\frac{\partial P}{\partial \epsilon} \frac{\mathrm{d} \epsilon}{\mathrm{d} T}
\end{array}
$$

which results in (eq 5)

$$
\alpha_{\mathrm{p}}=-\alpha_{1}-\alpha_{\mathrm{A}}+\alpha_{\mu}+\alpha_{\cos \theta}+\frac{\epsilon}{\epsilon+2} \alpha_{\epsilon}
$$

Here the first two terms describe the thermal volume expansion, consisting of change in thickness $l$ and sample area $A$. Since we measure the charge $\Delta Q$ rather than $\Delta P=\Delta(Q / A)$ (see discussion below), the term accounting for the change in area is set to zero.

The next expressions $\alpha_{\mu}$ and $\alpha_{\cos \theta}$ refer to the pyroelectric effect arising from a temperature-dependent dipole moment $\mu$ and temperature-dependent mean fluctuation angle $\theta . \quad \alpha_{\cos \theta}$ has been found to be the dominating pyroelectric component in $\mathrm{AlPO}_{4}-5$ zeolites loaded with $p \mathrm{NA}{ }^{8}$ In contrast, the guest molecules in PHTP inclusion compounds are incorporated in the host lattice at fixed crystallographic positions, which inhibits torsional fluctuations to a high degree. Hence we will also neglect $\alpha_{\cos \theta}$ in the following discussion.



Figure 2. Drawing of two AD guest molecules [1-(4-nitrophenyl)piperazine] as part of a dipole chain. $\mu_{1}$ is the dipole moment of one molecule and $\mu_{2}$ is the dipole moment of the H-bond between the two molecules.

The meaning of $\alpha_{\mu}$ will be illustrated by assuming that the total dipole moment $\mu$ may be represented by a simple vector sum of the two opposite dipole moments $\mu_{1}$ and $\mu_{2}$ according to Figure 2. The individual dipole moments are proportional to their length $l_{1}$ and $l_{2}$ and to their temperature dependence, which is given by the corresponding linear expansion coefficients $\alpha_{11}$ and $\alpha_{12}$, respectively. Using these microscopic quantities, one can derive eq 6 :

$$
\alpha_{\mu}=\frac{\alpha_{11} l_{1}-\alpha_{12} l_{2}}{l_{1}+l_{2}}
$$

which might become either positive or negative, depending on the weighted expansion coefficients $\alpha_{11}$ and $\alpha_{12}$. Assuming that the modulus of the H-bonds $\left(\mu_{2}\right)$ is small compared to the covalently bonded NPP group, so that $\alpha_{11} \ll \alpha_{12}\left(\alpha_{1}=\left(\alpha_{11} l_{1}+\right.\right.$ $\left.\alpha_{12} l_{2}\right) /\left(l_{1}+l_{2}\right)$ ), one finds $\alpha_{\mu} \cong-\alpha_{1}$, resulting in eq 7:

$$
\alpha_{p}=-2 \alpha_{1}+\frac{\epsilon}{\epsilon+2} \alpha_{\epsilon}
$$

The meaning of the coefficient $\alpha_{\epsilon}$ is closely related to the molecular origin of the electrical susceptibility $\chi_{\mathrm{el}}=\epsilon-1$. Since the thermal angular vibrations of the NPP molecules around their mean position are negligible, no significant orientational polarizability has to be considered. Hence the total polarizability is assumed to be both electronic and atomic in nature, and the density $N / V$ and dielectric constant are related by the Clausius-Mossotti equation:

$$
\frac{(\epsilon-1) V}{(\epsilon+2) N}=\text { const. }
$$

from which one can derive eq 9 in a straightforward way:

$$
\alpha_{\epsilon}=\frac{(\epsilon+2)(\epsilon-1)}{3 \epsilon} \alpha_{1}
$$

Combination of eqs 7 and 9 then yields an expression for $\alpha_{p}$ in which only geometrical changes due to thermal length expansion are responsible for the pyroelectric effect:

$$
\alpha_{\mathrm{p}}=-\frac{\epsilon+5}{3} \alpha_{1}
$$

Pyroelectric Response Equation. The link between the microscopically established quantity $\alpha_{\mathrm{p}}$, a local spatial temperature change $\partial T(x, \omega) / \partial t$, and the pyroelectric current $I(\omega)$ is given by eq 1 . Inserting eqs 9 and 10 in the first term of eq 1 results in

$$
I_{\mathrm{p}}(\omega)=-\alpha_{1} \frac{\epsilon+2}{3 \epsilon} \frac{A}{l} \int_{0}^{l} P^{*}(x) \frac{\partial T(x, \omega)}{\partial t} \mathrm{~d} x
$$

Analogous to the definition of the "true" pyroelectric coefficient, it seems useful at this point to introduce a modified pyroelectric 
coefficient $p^{*}$ :

$$
p(x)^{*}=-\alpha_{1} \frac{\epsilon+2}{3 \epsilon} P^{*}(x)
$$

Inserting the definition for $P^{*}(x)$ results in the expression for the modified pyroelectric coefficient, which will be compared with the experimental value (after calculating with values for $\alpha_{1}, \epsilon$, and $\left.P_{0}\right)$ :

$$
p^{*}=-\alpha_{1} \frac{(\epsilon+2)^{2}}{9 \epsilon} P_{0}
$$

We have stated before that eq 1 was originally derived for a strict one-dimensional electric field oriented parallel to the heat transport direction, as found typically in thin films or plates. Equations 1 and 11 also remain valid if only a part of the sample area is (homogeneously) heated, since the nonactive part acts as a capacitor in parallel to the active sample. In other words, as long as the field within the dielectric is parallel, all contributions arising from a different lateral position $\{y, z\}$ add up.

Let us consider now a needle-like crystal of thickness $l$ whose end faces are covered by metal electrodes. It is obvious that the electric field $E$ generated by polarization charges at position $\{x, y, z\}$ looks rather like that of two point charges than that of a parallel plate capacitor. Consequently, these electrodes do not "see" the complete field of the polarization charges enclosed, resulting in a pyroelectric response depending on the position of the inner charges relative to the electrodes.

To circumvent this difficult electrostatic situation, we have replaced the end face electrodes by large field plates $\left(\sqrt{A_{\mathrm{el}}} \gg\right.$ $l$ ), which shield the sample over almost the complete space angle $\Omega$ and align the electrical field parallel in the neighborhood of the electrodes. Also the above requirement of "soft" electrodes in order to neglect the lateral thermal expansion of the sample is automatically fulfilled by the use of electrodes whose size exceeds that of the sample area several times.

A second argument that supports the validity of eqs 1 and 11 , even for needle-like samples, arises from the fact that the higher dielectric constant of the crystal is $\sim 2.5$ times the permittivity of the surroundings, resulting in guiding of the field along the crystal's axis.

Summarizing, we trust in the validity of eqs 1 and 11 , even for the rod-like crystals considered in this study.

\section{Materials and Experimental Details}

3.1. Materials. Two different AD disubstituted chromophores were investigated. The PHTP-NPP crystals [NPP: 1-(4nitrophenyl)piperazine] were grown by a previously described temperature difference method using a methyl ethyl ketone solution. ${ }^{1,2}$ Crystallization of PHTP-INBP crystals [INBP: 4-iodo-4'-nitrobiphenyl] was carried out in stirred solutions of purified paraldehyde by the controlled lowering of the temperature $\left(60{ }^{\circ} \mathrm{C} \rightarrow 48{ }^{\circ} \mathrm{C}\right)$, in order to keep supercooling to a minimum $(\Delta T<2-3 \mathrm{~K})$. Solutions $(1.5-2 \mathrm{~mL})$ were prepared by dissolving PHTP $(25-30 \mathrm{mg})$ and INBP $(3-4 \mathrm{mg})$ in paraldehyde.

Needle-shaped crystals of size $1-3 \mathrm{~mm}$ in length and $0.2-$ $0.5 \mathrm{~mm}$ in thickness were obtained. Their cross section was nearly hexagonal. The needle axis corresponds to the channel axis (denoted by 3, see Figure 1).

3.2. Experimental Details. The experimental setup is shown in Figure 3. An intensity-modulated laser diode (LISA HL25/

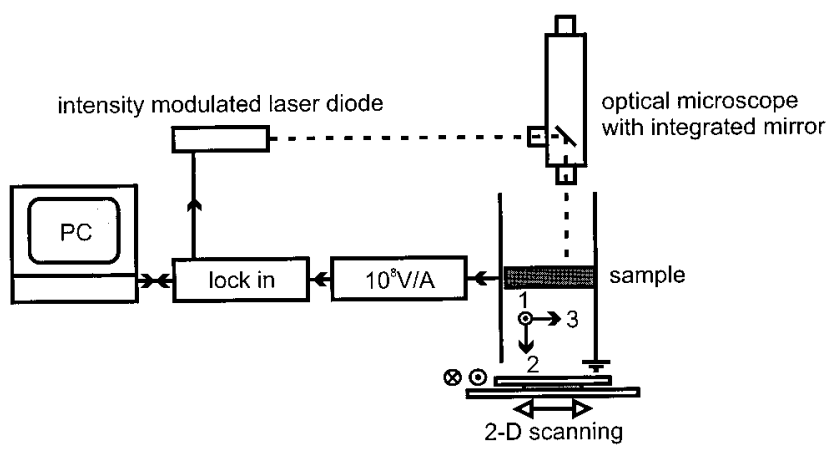

Figure 3. Experimental setup.

$\mathrm{M} 2,5 \mathrm{~mW}, \lambda=635 \mathrm{~nm}$ ) served as the heat source. The sample response was fed to a current amplifier (Keithley model 428, gain $10^{8} \mathrm{~V} / \mathrm{A}$ ). A lock-in amplifier (Stanford Research 850) provided the sinusoidal reference voltage for modulating the laser source and for the phase-sensitive analysis of the pyroelectric response. Modulation frequencies from $0.1 \mathrm{~Hz}$ to 10 $\mathrm{kHz}$ were used. Pyroelectric spectra were measured with a current sensitivity better than $10^{-14} \mathrm{~A}$. The entire data acquisition was under computer control.

The sample was placed between two plate electrodes (area: $1 \mathrm{~cm}^{2}$ ), contacted with conducting silver paste on the low (-) side, thermally and mechanically separated by an air gap (diameter $<100 \mu \mathrm{m}$ ) from the high $(+)$ side. For scanning the sample in the 1- and 3-direction the sample holder was mounted on a motorized displacement unit. Rotating the sample around the 3-axis enabled scanning along the 2-axis as well. Incorporating an optical microscope allowed inspection of the sample during placement in the sample holder and measurement. The minimum diameter of the focused laser spot was about 10 $\mu \mathrm{m}$. The typical scan increment was $20 \mu \mathrm{m}$ along the three axes. Dipping the sample into an ethanolic solution of a black dye extracted from a permanent marker (MarkAll supermarker permanent) produced a thin and homogeneous black absorption layer. Measurements were performed in air and at $25^{\circ} \mathrm{C}$. With this setup it was possible to obtain a highly resolved $3-\mathrm{D}$ picture of the pyroelectric response.

\section{Results and Discussion}

4.1. SPEM Measurements on PHTP-NPP Crystals. The SPEM images are shown in Figure 4. Images $a-d$ give the position-dependent pyroelectric response at a constant frequency of $415 \mathrm{~Hz}$ from four sides of the same crystal as sketched below the images. With a thermal diffusivity $K$ of $1.33 \times 10^{-7} \mathrm{~m}^{2}$ $\mathrm{s}^{-1}$ (ref 14), the thermal diffusion length $\lambda_{\text {th }}$ is $10 \mu \mathrm{m}$, which means that the heated surface layer is around $10 \mu \mathrm{m}$ thick. The step size was always $20 \mu \mathrm{m}$, which is above the lateral resolution limit of $15 \mu \mathrm{m}$. The red regions represent a positive signal, whereas blue is negative and white is zero. In all scans there are two regions of opposite pyroelectric current, which divide the crystal into two halves. Near the end faces, there is some response from reflections from the electrode, which hit the sample while the laser scans the electrode. The crystal shape is well mapped. In the lower half there is an intrusion caused by a second smaller crystal, which grew on the large crystal and was lost during sample preparation. Its position and shape can well be recognized in Figure 4c,d, while Figure 4a,b show only a slightly enhanced pyroelectric response at the corresponding places. This can be explained by a distortion in the local electric field distribution as well as by deviations from the thermal model. Between the highly polar regions, there are small areas that appear to be nonpolar. Performing a second 




a)

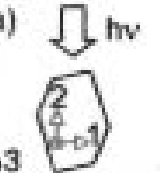

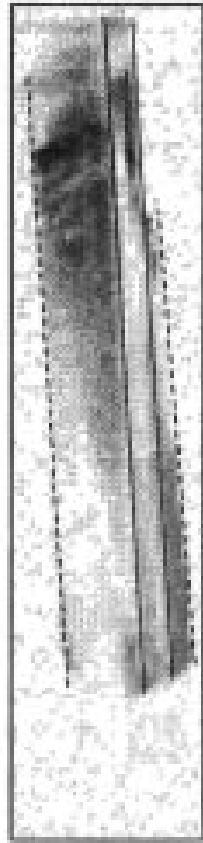

b)

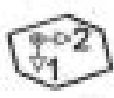

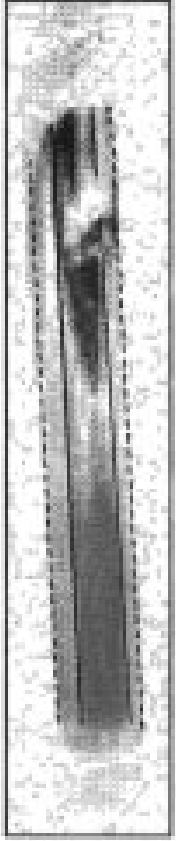

c)

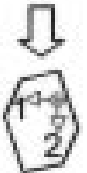



d)

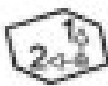



e)

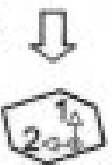

Figure 4. SPEM images of a PHTP-NPP single crystal. Position-dependent pyroelectric response at constant frequency from four sides, as sketched below the images. Step size: $20 \mu \mathrm{m}$. Red corresponds to a positive current while blue and white indicate a negative and zero response, respectively. (a-d) $f=415 \mathrm{~Hz}, \lambda_{\text {th }} \approx 10 \mu \mathrm{m}$. (e) $f=21 \mathrm{~Hz}, \lambda_{\text {th }} \approx 40 \mu \mathrm{m}$.



a)



b)

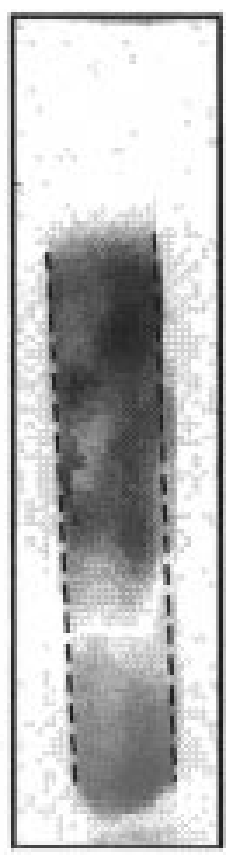

c)

Figure 5. SPEM images of the same PHTP-NPP crystal as in Figure 4 after thinning by an ethanol/methyl ethyl ketone solution. Step-size: $20 \mu \mathrm{m}$. (a) $f=415 \mathrm{~Hz}$ [same side as Figure 4d]. (b) $f=415 \mathrm{~Hz}$, (c) $f=21 \mathrm{~Hz}[(\mathrm{~b}, \mathrm{c})$ : same side as Figure $4 \mathrm{~b}]$.

sample scan with a significant larger thermal diffusion length ( $\lambda_{\text {th }} \approx 40 \mu \mathrm{m}, f=21 \mathrm{~Hz}$ ), we find that most of these white spots disappear, indicating that these nonpolar regions are around $10 \mu \mathrm{m}$ thick (Figure 4e).

Figure 5 shows the same crystal after a procedure of thinning it by an ethanol/methyl ethyl ketone solution. Figure $5 \mathrm{a}$ is a scan at $415 \mathrm{~Hz}$ of the same side as in Figure 4d. Figures 5b) $(415 \mathrm{~Hz})$ and $5 \mathrm{c}(21 \mathrm{~Hz})$ represent the same side as in Figure 4b). This inner part of the crystal behaves in nearly the same manner as the outer part. The $21 \mathrm{~Hz}$ scan shows two homogeneous polarized regions with a sharp interface.

To estimate a maximum value for the pyroelectric coefficient, one has to consider the response equation for a thin, homogenously polarized sample, which consists only of the second term in eq 1 . With the modulated laser intensity $H(t)=H_{0}$ $\sin (\omega t)$ and the temperature

$$
T(t)=T_{0}+\int_{0}^{t} \frac{H(t)}{A l_{3} c_{\mathrm{p}} \rho} \mathrm{d} t
$$

the real part of the complex amplitude of the pyroelectric current is

$$
\operatorname{Re}\left\{I_{\sim}\right\}=p_{\exp } A \frac{\mathrm{d} T}{\mathrm{~d} t}=\frac{p_{\exp } H_{0}}{l_{3} c_{\mathrm{p}} \rho}
$$

At a modulation amplitude of the laser intensity of $H_{0}=0.4$ $\mathrm{mW}$ we found a maximum pyroelectric response of $\operatorname{Re}\left\{I_{\sim}\right\}=$ $-0.2 \mathrm{pA}$ for a crystal length of $l_{3}=2 \times 10^{-3} \mathrm{~m}$. For a product of specific heat and density of PHTP-NPP, $c_{p} \rho \approx 1.5 \times 10^{6} \mathrm{~J}$ $\mathrm{m}^{-3} \mathrm{~K}^{-1}$ (ref 15) and eq 15, a pyroelectric coefficient of

$$
p_{\exp }=\frac{\operatorname{Re}\left\{I_{\sim}\right\} l_{3} c_{\mathrm{p}} \rho}{H_{0}} \approx-1.5 \mu \mathrm{C} \mathrm{m}^{-2} \mathrm{~K}^{-1}
$$

is obtained. It may represent a maximum value for the analyzed PHTP-NPP crystals. This estimated value is about 1 order of 


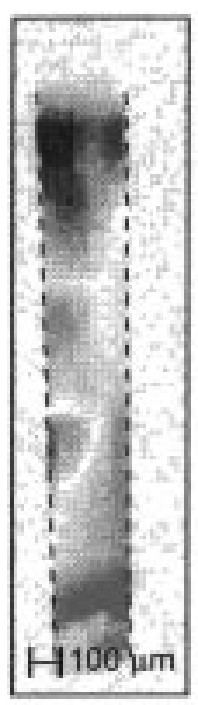

a)



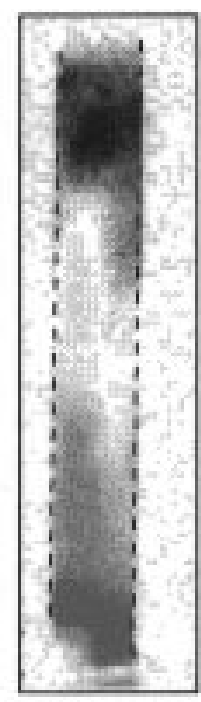

b)





c)



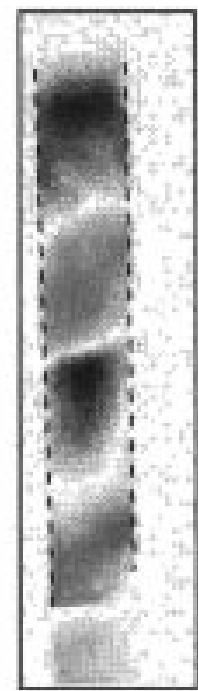

d)



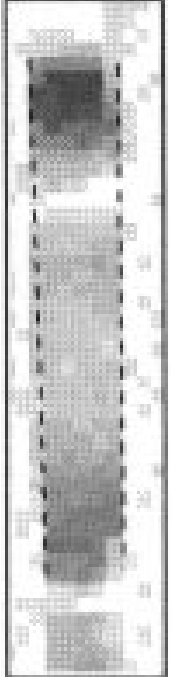

e)

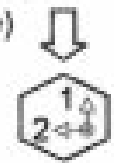

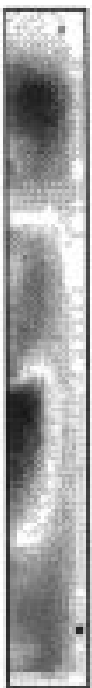

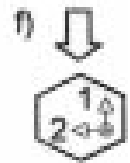

Figure 6. (a-e) SPEM images of a PHTP-INBP crystal. (a-d) Step size: $20 \mu \mathrm{m}, f=21 \mathrm{~Hz}$. (e) Step size: $50 \mu \mathrm{m}, f=1 \mathrm{~Hz}$. (f) Pyroelectric spectra taken along the needle axis close to the crystal edge [same view as for (d) and (e)]. Note that here the horizontal axis corresponds to a logarithmically spaced frequency axis [left, $415 \mathrm{~Hz}$; right, $0.1 \mathrm{~Hz}]$.

magnitude lower than for $\operatorname{PVDF}\left(p \approx-25 \mu \mathrm{C} \mathrm{m}{ }^{-2} \mathrm{~K}^{-1}\right){ }^{16}$ This experimental value can be compared with a calculated value from eq 13. With $\alpha_{1}=6 \times 10^{-5} \mathrm{~K}^{-1}$ (calorimetric measurement, $\alpha_{3} \approx 5 \times 10^{-5} \mathrm{~K}^{-1}$, ref 17 ; from X-ray data, $\alpha_{3} \approx 7 \times$ $10^{-5} \mathrm{~K}^{-1}$, ref 18$), \epsilon=2.3($ ref 19$), P_{0} \approx 10 \mathrm{mC} / \mathrm{m}^{2}(N / V=$ $1 / 2000 \AA^{3}=5 \times 10^{26} \mathrm{~m}^{-3}, \mu=7 \mathrm{D}=7 \times 3.34 \times 10^{-30} \mathrm{C} \mathrm{m}$, ref $20,\langle\cos \theta\rangle \approx 1)$, the calculated value is $p^{*}=-0.54 \mu \mathrm{C}$ $\mathrm{m}^{-2} \mathrm{~K}^{-1}$, which is of the same order of magnitude as the maximum experimental value.

4.2. SPEM Measurements and Position-Dependent Pyroelectric Spectra for PHTP-INBP Crystals. The positiondependent pyroelectric response at constant frequency of $21 \mathrm{~Hz}$ of four sides of the same sample is shown in Figure $6 a-d$ ). The 2,3-side (Figure 6b) looks quite the same as the PHTPNPP crystal (section 4.1). The other three sides show the response of four regions with alternating sign of the current. Probably the crystal grew from more than one seed. Figure 6e is a scan of the same side as Figure $6 \mathrm{~d}$ but with $1 \mathrm{~Hz}$ (and a scan increment of $50 \mu \mathrm{m}$ ), which means that the whole cross section of the sample is isothermally heated [(case d) in Figure 2]. On average, the crystal consists of two regions yielding an opposite pyroelectric current response. Figure 6f shows the position-dependent pyroelectric frequency spectra from a line scan in the middle of the same side as in Figures $6 \mathrm{~d}$ and $6 \mathrm{e}$. For high frequencies the lateral polarization distribution near the surface is probed, whereas for low frequencies the polarization distribution in the length direction is the main contribution to the signal. The frequency spectra deliver a kind of depth profile of the sample with the restriction that the signal is always an average over the whole heated area. The combination of 1-D frequency spectra with 2-D scans at constant frequency leads to 3-D information about the polarization distribution.

Summarizing our first two cases we have analyzed here, we conclude that in the regions where the pyroelectric response is a maximum, all dipoles are aligned parallel. This is in agreement with the crystal structure determination of PHTPNPP, which reports a polar space group. ${ }^{5}$ From the current direction we can conclude that both types of crystals are negatively charged at their ends, compensated by positive external charges. This means that the A (nitro) groups are pointing toward the capping faces. These findings are in complete agreement with the predictions of Markov's theory for the selective mechanism of attachment of dipoles at the capping faces. ${ }^{4,5}$

\section{Conclusions}

The extension of the simple 1-D pyroelectric scanning technique to 2-D has proven to be a useful tool for mapping the polarization distribution in inhomogeneous crystals. By this new technique we have demonstrated that PHTP-AD crystals grow as $180^{\circ}$ twins, and supported by the theoretical considerations of the origin of the pyroelectic effect in these materials, we can assume a negative pyroelectric coefficient along the channel direction. This allows us than to conclude that the acceptor groups predominantly cover the growing c-faces. Scans at high frequency furthermore confirmed the hourglass-like polarity development (Figures 4-6) predicted by the Markov growth model. Data at a higher resolution for the more detailed structure of the polarity development can be expected from 2-D scanning experiments where crystals are stepwise reduced in thickness by fine polishing. This work is in progress.

Acknowledgment. This work was supported by the Swiss National Science Foundation [NFP 36 (4036-0439932) and NF (20-4316.95)]. We thank Dr. O. König of Molecular Simulations Ltd., Cambridge, U.K., for the calculation of the dipole moment, Prof. R. Giovanoli (Berne) for the DSC measurements, and P. Reber for the synthesis and purification of the compounds employed in this work.

\section{References and Notes}

(1) Hulliger, J.; König, O.; Hoss, R. Adv. Mater. 1995, 7, 719.

(2) Hoss, R.; König, O.; Kramer-Hoss, V.; Berger, U.; Rogin, P.; Hulliger, J. Angew. Chem., Ed. Int. Engl. 1996, 35, 1664.

(3) König, O.; Hulliger, J. Mol. Cryst. Liq. Cryst. Sect. B: Nonlinear Opt. 1997, 17, 127. 
(4) Hulliger, J.; Rogin, P.; Quintel, A.; Rechsteiner, P.; König, O.; Wübbenhorst, M. Adv. Mater. 1997, 9, 677.

(5) König, O.; Bürgi, H.-B.; Armbruster, Th.; Hulliger, J.; Weber, Th. J. Am.Chem. Soc. 1997, 119, 10632.

(6) Hadni, A.; Bassia, J.M.; Gerbaux, X.; Thomas, R. Appl. Opt. 1976, $15,2150$.

(7) Yilmaz, S.; Bauer, S.; Wirges, W.; Gerhard-Multhaupt, R. Appl. Phys. Lett. 1993, 63, 1724

(8) Marlow, F.; Wübbenhorst, M.; Caro, J. J. Phys. Chem. 1994, 98 (47), 12315.

(9) Patil, A. A.; Curtin, D. Y.; Paul, I. C. J. Am. Chem. Soc. 1985, 107,726 .

(10) Collins, R. E. J. Appl. Phys. 1976, 47, 4804

(11) Lang, S. B. Ferroelectrics 1991, 118, 343.

(12) Lang, S. B. Ferroelectrics 1990, 106, 269.

(13) Bossard, C.; Sutter, K.; Prêtre, P.; Hulliger, J.; Flörsheimer, M.; Kaatz, P.; Günter, P. Organic Nonlinear Optical Materials; Gordon and Breach: Basel, 1995
(14) Wübbenhorst, M.; Quintel, A.; Hulliger, J. In preparation.

(15) The density of PHTP-NPP is $\rho \approx 1.14 \times 10^{3} \mathrm{~kg} \mathrm{~m}^{-3}$ (calculated from X-ray data ${ }^{5}$ ). The heat capacity of PHTP-NPP was measured with a Perkin Elmer differential scanning calorimeter (DSC) to be $c_{\mathrm{p}} \approx 1.3 \times 10^{3}$ $\mathrm{J} \mathrm{kg}^{-1} \mathrm{~K}^{-1}$

(16) Technical Data, Piezoelectric SOLEF PVDF polyvinylidene fluoride films, Solvay \& Cie., Rue du Prince Albert 44, 1050 Bruxelles, Belgium.

(17) The length coefficient of thermal expansion was measured with a dynamic mechanical analyzer (DMA7e Perkin Elmer).

(18) The length coefficient of thermal expansion can be estimated by $\alpha_{c}=(1 / \bar{c})(\Delta c / \Delta T)$, with $c(300 \mathrm{~K})=4.797 \AA$ and $c(100 \mathrm{~K})=4.730 \AA$, known from X-ray diffraction, ${ }^{5} \bar{c}=(c(300 \mathrm{~K})+c(100 \mathrm{~K})) / 2$

(19) The permittivity $\epsilon$ was measured with a dielectric bridge $(1 \mathrm{kHz}$ precision bridge AH 2500, Andeen Hageling).

(20) The dipole moment of NPP was obtained from the semiempirical quantum mechanics package MOPAC (MNDO approximation) using the Cerius2 software program from Molecular Simulations Ltd., Cambridge, U.K. 